\title{
Multiple positive solutions of a nonlinear fourth order periodic boundary value problem
}

\author{
by Lingbin Kong (Anda) and Daqing JiAng (Changchun)
}

\begin{abstract}
The fourth order periodic boundary value problem $u^{(4)}-m^{4} u+F(t, u)=0$, $0<t<2 \pi$, with $u^{(i)}(0)=u^{(i)}(2 \pi), i=0,1,2,3$, is studied by using the fixed point index of mappings in cones, where $F$ is a nonnegative continuous function and $0<m<1$. Under suitable conditions on $F$, it is proved that the problem has at least two positive solutions if $m \in(0, M)$, where $M$ is the smallest positive root of the equation $\tan m \pi=-\tanh m \pi$, which takes the value 0.7528094 with an error of $\pm 10^{-7}$.
\end{abstract}

1. Introduction. This paper deals with the fourth order periodic boundary value problem

$$
\begin{cases}u^{(4)}-m^{4} u+F(t, u)=0, & 0<t<2 \pi \\ u^{(i)}(0)=u^{(i)}(2 \pi), & i=0,1,2,3\end{cases}
$$

where $0<m<1$ and $F:[0,2 \pi] \times[0, \infty) \rightarrow[0, \infty)$ is a nonnegative continuous function.

Recently, the periodic boundary value problems have been studied extensively (see [1-2, 4-7] and references therein). In [1], A. Cabada studied a fourth order periodic boundary value problem similar to (1.1), using a generalized method of upper and lower solutions and developing the monotone iterative technique in the presence of upper and lower solutions, but he did not study the multiplicity of the solutions.

The purpose of this paper is to study the existence of multiple positive solutions to the problem (1.1) by using the fixed point index of mappings in cones. Our method is different from [1] and yields a multiplicity result for positive solutions.

The following hypotheses are adopted in this paper, depending on various circumstances:

1991 Mathematics Subject Classification: 34B10, 34B15, 34C25.

Key words and phrases: nonlinear periodic boundary value problem, multiple positive solutions, cone, fixed point index. 
(H1) There exists a $p>0$ such that $0 \leq u \leq p$ implies $F(t, u)<\lambda p$, where

$$
\lambda=\frac{1}{2 \pi G(\pi, m)}, \quad G(\pi, m)=\frac{1}{4 m^{3}}\left(\frac{1}{\sinh m \pi}+\frac{1}{\sin m \pi}\right) .
$$

(H2) There exists a $p>0$ such that $\sigma p \leq u \leq p$ implies $F(t, u)>(\lambda / \sigma) p$, where

$$
\sigma=\frac{\left(e^{2 m \pi}-1\right) \cos m \pi+\left(e^{2 m \pi}+1\right) \sin m \pi}{e^{2 m \pi}+2 e^{m \pi} \sin m \pi-1} .
$$

We call a function $u(t)$ a positive solution of (1.1) if it satisfies:

(1) $u \in C^{3}[0,2 \pi] \cap C^{4}(0,2 \pi), u^{(i)}(0)=u^{(i)}(2 \pi), i=0,1,2,3$, and $u(t)>$ 0 for all $t \in(0,2 \pi)$, and

(2) the equality $u^{(4)}-m^{4} u=-F(t, u)$ holds for all $t \in(0,2 \pi)$.

The main result of this paper is as follows.

Theorem 1. If $m \in(0, M)$, then the problem (1.1) has at least two positive solutions $u_{1}$ and $u_{2}$ satisfying $0<\left\|u_{1}\right\|<p<\left\|u_{2}\right\|$ provided that

(I) the condition (H1) holds and

$$
\lim _{u \rightarrow 0} \min _{t \in[0,2 \pi]} \frac{F(t, u)}{u}>\frac{\lambda}{\sigma^{2}}, \quad \lim _{u \rightarrow \infty} \min _{t \in[0,2 \pi]} \frac{F(t, u)}{u}>\frac{\lambda}{\sigma^{2}}, \quad \text { or }
$$

(II) the condition (H2) holds and

$$
\lim _{u \rightarrow 0} \max _{t \in[0,2 \pi]} \frac{F(t, u)}{u}<\lambda, \quad \lim _{u \rightarrow \infty} \max _{t \in[0,2 \pi]} \frac{F(t, u)}{u}<\lambda,
$$

where $M$ is the smallest positive root of the equation $\tan m \pi=-\tanh m \pi$, and $\lambda$ is given by (1.2).

The following theorem will be used in our proof (see [3]).

Theorem 2. Let $E$ be a Banach space, and $K \subseteq E$ a cone in $E$. For $p>0$, define $K_{p}=\{u \in K:\|u\| \leq p\}$. Assume that $\Phi: K_{p} \rightarrow K$ is a compact map such that $\Phi u \neq u$ for $u \in \partial K_{p}=\{u \in K:\|u\|=p\}$.

(i) If $\|u\| \leq\|\Phi u\|$ for $u \in \partial K_{p}$, then $i\left(\Phi, K_{p}, K\right)=0$.

(ii) If $\|u\| \geq\|\Phi u\|$ for $u \in \partial K_{p}$, then $i\left(\Phi, K_{p}, K\right)=1$.

2. Proof of Theorem 1. As shown in [1], problem (1.1) is equivalent to the integral equation

$$
u(t)=\int_{0}^{2 \pi} G(t, s, m) F(s, u(s)) d s
$$


where

$$
\begin{aligned}
G(t, s, m) & =G(|t-s|, m) \\
& = \begin{cases}\frac{f(t-s)+g(t-s)}{4 m^{3}\left(e^{m \pi}-e^{-m \pi}\right)^{2}(1-\cos 2 m \pi)}, & 0 \leq s \leq t \leq 2 \pi, \\
\frac{f(2 \pi+t-s)+g(2 \pi+t-s)}{4 m^{3}\left(e^{m \pi}-e^{-m \pi}\right)^{2}(1-\cos 2 m \pi)}, & 0 \leq t \leq s \leq 2 \pi,\end{cases}
\end{aligned}
$$

and

$$
\begin{aligned}
& f(t)=\left(e^{m \pi}-e^{-m \pi}\right)^{2}(\sin m t+\sin m(2 \pi-t)), \\
& g(t)=\left(e^{m t}-e^{-m t}+e^{m(2 \pi-t)}-e^{-m(2 \pi-t)}\right)(1-\cos 2 m \pi) .
\end{aligned}
$$

Lemma 1. If $m \in(0,1)$, then the function $G(t, m)$ in the interval $[0,2 \pi]$ attains its minimum for $t=0$ and its maximum for $t=\pi$.

Proof. Let $w(t)=f(t)+g(t)$. Since $w(2 \pi-t)=w(t)$, it suffices to consider the function $w$ in the interval $[0, \pi]$.

If $m \in(0,1 / 2]$, then by a direct computation, we get $w^{(4)}(t)>0$ in $[0, \pi]$ and $w^{\prime \prime \prime}(\pi)=0$, and hence $w^{\prime \prime \prime}(t) \leq 0$ in $[0, \pi]$. Thus, $w^{\prime}(t)$ is a concave function in $[0, \pi]$. Moreover, since $w^{\prime}(0)=0$ and $w^{\prime}(\pi)=0$, we have $w^{\prime}(t) \geq 0$ in $[0, \pi]$. Therefore, $w(t)$ is nondecreasing in $[0, \pi]$.

In [1] it is proved that, if $m \in(1 / 2,1)$, then the unique root of $f$ in $[0, \pi]$ is $\frac{2 m-1}{2 m} \pi$, and $w(t)$ is nondecreasing in $\left[0, \frac{2 m-1}{2 m} \pi\right]$. We claim that, if $m \in(1 / 2,1)$, then $w(t)$ is also nondecreasing in $\left[\frac{2 m-1}{2 m} \pi, \pi\right]$. In fact, it is not difficult to show that $w^{(4)}(t)>0$ in $\left[\frac{2 m-1}{2 m} \pi, \pi\right]$ and $w^{\prime \prime \prime}(\pi)=0$, so $w^{\prime \prime \prime}(t) \leq 0$ in $\left[\frac{2 m-1}{2 m} \pi, \pi\right]$, and hence $w^{\prime}(t)$ is concave in $\left[\frac{2 m-1}{2 m} \pi, \pi\right]$. Moreover, since $w^{\prime}\left(\frac{2 m-1}{2 m} \pi\right)>0$ and $w^{\prime}(\pi)=0$, we have $w^{\prime}(t) \geq 0$ in $\left[\frac{2 m-1}{2 m} \pi, \pi\right]$. This shows our claim.

To sum up, the function $w(t)$ attains its minimum in $[0,2 \pi]$ at $t=0$ and its maximum at $t=\pi$, and so does $G(t, m)$. The proof is complete.

By Lemma 1, the greatest value of $m$ for which $G(t, m)$ is positive in $[0,2 \pi]$ will be the smallest positive zero of the expression

$$
w(0)=\left(e^{2 m \pi}-e^{-2 m \pi}\right)(1-\cos 2 m \pi)+\left(e^{m \pi}-e^{-m \pi}\right)^{2} \sin 2 m \pi .
$$

This expression is zero if and only if either $m \in \mathbb{N}$ or

$$
\tan m \pi=-\tanh m \pi \text {. }
$$

The smallest positive root of (2.5), which we denote by $M$, takes a value of 0.7528094 with an error of $\pm 10^{-7}$. This is the unique root in $(0,1)$ (see [1]).

Let $m \in(0, M) \subset(0,1)$. Then $G(0, m)>0$. Define the mapping $\Phi: C[0,2 \pi] \rightarrow C[0,2 \pi]$ by

$$
(\Phi u)(t):=\int_{0}^{2 \pi} G(t, s, m) F(s, u(s)) d s .
$$


It is obvious that $\Phi$ is completely continuous. We define a cone in the Banach space $C[0,2 \pi]$ by

$$
K:=\left\{u \in C[0,2 \pi]: u(t) \geq 0 \text { for all } t \text { and } \min _{t \in[0,2 \pi]} u(t) \geq \sigma\|u\|\right\},
$$

where $\|u\|=\sup _{t \in[0,2 \pi]}|u(t)|$ and $\sigma$ is given by (1.3).

Lemma 2. $\Phi(K) \subset K$.

Proof. Lemma 1 implies

$$
\sigma=\frac{G(0, m)}{G(\pi, m)} \leq \frac{G(t, s, m)}{G(\pi, m)} \leq 1,
$$

and hence for $u \in K$ we have

$$
\begin{aligned}
\min _{t \in[0,2 \pi]}(\Phi u)(t) & =\min _{t \in[0,2 \pi]} \int_{0}^{2 \pi} G(t, s, m) F(s, u(s)) d s \\
& \geq \sigma \int_{0}^{2 \pi} G(\pi, m) F(s, u(s)) d s \\
& \geq \sigma \max _{t \in[0,2 \pi]} \int_{0}^{2 \pi} G(t, s, m) F(s, u(s)) d s=\sigma\|\Phi u\| .
\end{aligned}
$$

This shows that $\Phi(K) \subset K$.

Now we prove the first part of Theorem 1. Since

$$
\lim _{u \rightarrow 0} \min _{t \in[0,2 \pi]} \frac{F(t, u)}{u}>\frac{\lambda}{\sigma^{2}},
$$

there exists a $0<r<p$ such that $F(t, u)>\left(\lambda / \sigma^{2}\right) u$ for $0 \leq u \leq r$. For $u \in \partial K_{r}=\{u \in K:\|u\|=r\}$, we have

$$
\begin{aligned}
\|\Phi u\| & =\max _{t \in[0,2 \pi]} \int_{0}^{2 \pi} G(t, s, m) F(s, u(s)) d s>\frac{\lambda}{\sigma} G(\pi, m) \int_{0}^{2 \pi} u(s) d s \\
& \geq 2 \pi \lambda G(\pi, m)\|u\|=\|u\|,
\end{aligned}
$$

i.e. $\|\Phi u\|>\|u\|$ for $u \in \partial K_{r}$, and hence Theorem 2 implies

$$
i\left(\Phi, K_{r}, K\right)=0 .
$$

In much the same way, we may prove that there exists an $R>p$ such that $\|\Phi u\|>\|u\|$ for $u \in \partial K_{R}$ by using $\lim _{u \rightarrow \infty} \min _{t \in[0,2 \pi]} F(t, u) / u>\lambda / \sigma^{2}$. Hence Theorem 2 again implies

$$
i\left(\Phi, K_{R}, K\right)=0 .
$$


On the other hand, by (H1), for $u \in \partial K_{p}$ we have

$$
\begin{aligned}
\|\Phi u\| & =\max _{t \in[0,2 \pi]} \int_{0}^{2 \pi} G(t, s, m) F(s, u(s)) d s \leq G(\pi, m) \int_{0}^{2 \pi} F(s, u(s)) d s \\
& <2 \pi \lambda p G(\pi, m)=\|u\|,
\end{aligned}
$$

i.e. $\|\Phi u\|<\|u\|$ for $u \in \partial K_{p}$. It follows from Theorem 2 that

$$
i\left(\Phi, K_{p}, K\right)=1 \text {. }
$$

Now, the additivity of the fixed point index and (2.7)-(2.9) together imply

$$
i\left(\Phi, K_{p} \backslash \stackrel{\circ}{K}_{r}, K\right)=1, \quad i\left(\Phi, K_{R} \backslash \stackrel{\circ}{K}_{p}, K\right)=-1 .
$$

Consequently, $\Phi$ has a fixed point $u_{1}$ in $K_{p} \backslash \stackrel{\circ}{K}_{r}$, and a fixed point $u_{2}$ in $K_{R} \backslash \stackrel{\circ}{K}_{p}$. Both are positive solutions of the problem (1.1). It is obvious that $0<\left\|u_{1}\right\|<p<\left\|u_{2}\right\|$. This completes the proof of the first part.

We now prove the second part of Theorem 1. Since

$$
\lim _{u \rightarrow 0} \max _{t \in[0,2 \pi]} \frac{F(t, u)}{u}<\lambda,
$$

there exists a $0<r<p$ such that $F(t, u)<\lambda u$ for $0 \leq u \leq r$. For $u \in \partial K_{r}$, we have

$$
\begin{aligned}
\|\Phi u\| & =\max _{t \in[0,2 \pi]} \int_{0}^{2 \pi} G(t, s, m) F(s, u(s)) d s \leq G(\pi, m) \int_{0}^{2 \pi} F(s, u(s)) d s \\
& <2 \pi \lambda G(\pi, m)\|u\|=\|u\| .
\end{aligned}
$$

This shows that $\|\Phi u\|<\|u\|$ for $u \in \partial K_{r}$, and hence Theorem 2 implies

$$
i\left(\Phi, K_{r}, K\right)=1 \text {. }
$$

Similarly, we may prove that there exists $R>p$ such that $\|\Phi u\|<\|u\|$ for $u \in \partial K_{R}$ by using $\lim _{u \rightarrow \infty} \max _{t \in[0,2 \pi]} F(t, u) / u<\lambda$. Hence Theorem 2 again implies

$$
i\left(\Phi, K_{R}, K\right)=1 .
$$

In addition, since $\min _{t \in[0,2 \pi]} u(t) \geq \sigma\|u\|=\sigma p$ for $u \in \partial K_{p}$, using (H2) we have, for such $u$,

$$
\begin{aligned}
\|\Phi u\| & =\max _{t \in[0,2 \pi]} \int_{0}^{2 \pi} G(t, s, m) F(s, u(s)) d s \geq \sigma G(\pi, m) \int_{0}^{2 \pi} F(s, u(s)) d s \\
& >2 \pi \lambda p G(\pi, m)=\|u\|,
\end{aligned}
$$

i.e. $\|\Phi u\|>\|u\|$ for $u \in \partial K_{p}$. Thus, Theorem 2 implies

$$
i\left(\Phi, K_{p}, K\right)=0 \text {. }
$$


As before, (2.10)-(2.12) show that $\Phi$ has two positive fixed points, which means that the problem (1.1) has two positive solutions. The proof is complete.

\section{References}

[1] A. Cabada, The method of lower and upper solutions for second, third, fourth and higher order boundary value problems, J. Math. Anal. Appl. 185 (1994), 302-320.

[2] A. Cabada and J. J. Nieto, A generalization of the monotone iterative technique for nonlinear second-order periodic boundary value problems, ibid. 151 (1990), 181-189.

[3] L. H. Erbe, S. C. Hu and H. Y. W ang, Multiple positive solutions of some boundary value problems, ibid. 184 (1994), 640-648.

[4] W. J. Gao and J. Y. Wang, On a nonlinear second order periodic boundary value problem with Carathéodory functions, Ann. Polon. Math. 62 (1995), 283-291.

[5] D. Q. Jiang and J. Y. Wang, A generalized periodic boundary value problem for the one-dimensional p-Laplacian, ibid. 65 (1997), 265-270.

[6] V. Šeda, J. J. Nieto and M. Gera, Periodic boundary value problems for nonlinear higher order ordinary differential equations, Appl. Math. Comput. 48 (1992), 71-82.

[7] M. X. Wang, A. Cabada and J. J. Nieto, Monotone method for nonlinear second order periodic boundary value problems with Carathéodory functions, Ann. Polon. Math. 58 (1993), 221-235.

Department of Mathematics Daqing Petroleum Institute Anda 151400, Heilongjiang, P.R. China E-mail:wxw@dqpi.cnpc.com.cn
Department of Mathematics Northeast Normal University Changchun 130024, P.R. China E-mail: sxxi@ivy.nenu.edu.cn 